\title{
The ENPP1 K121Q polymorphism determines individual susceptibility to the insulin-sensitising effect of lifestyle intervention
}

\author{
K. Müssig • M. Heni • C. Thamer • K. Kantartzis • \\ F. Machicao • N. Stefan • A. Fritsche • H.-U. Häring • \\ H. Staiger
}

Received: 14 August 2009 / Accepted: 2 November 2009/Published online: 29 November 2009

(C) Springer-Verlag 2009

\begin{abstract}
Aims/hypothesis The K121Q (rs1044498) single nucleotide polymorphism (SNP) in the ENPP1 gene has shown association with insulin resistance and type 2 diabetes in various ethnic populations. We hypothesised that K121Q may predict the success of lifestyle intervention in terms of improvement of insulin sensitivity.

Methods We genotyped 1,563 participants with an increased risk of type 2 diabetes for K121Q and performed correlational analyses with anthropometric data and variables of insulin sensitivity. For metabolic characterisation, all participants underwent an OGTT. A subgroup of 506 participants additionally underwent a euglycaemic-hyperinsulinaemic clamp. In 342 participants, metabolic traits and anthropometric data were re-evaluated after a 9 month lifestyle intervention.

Results In the overall cohort, K121Q was not associated with measures of obesity, indices of glucose tolerance
\end{abstract}

Electronic supplementary material The online version of this article (doi:10.1007/s00125-009-1612-4) contains supplementary material, which is available to authorised users.

K. Müssig $\cdot$ M. Heni $\cdot$ C. Thamer $\cdot$ K. Kantartzis $\cdot$ F. Machicao $\cdot$

N. Stefan $\cdot$ A. Fritsche $\cdot$ H.-U. Häring $(\bowtie) \cdot H$. Staiger

Department of Internal Medicine IV (Endocrinology, Diabetology,

Angiology, Nephrology, and Clinical Chemistry),

University Hospital of Tübingen,

Otfried-Müller-Str. 10,

72076 Tübingen, Germany

e-mail: hans-ulrich.haering@med.uni-tuebingen.de

\section{A. Fritsche}

Division of Nutritional and Preventive Medicine,

Department of Internal Medicine,

University Hospital of Tübingen,

Tübingen, Germany during OGTT and insulin sensitivity estimated from the OGTT or derived from a euglycaemic-hyperinsulinaemic clamp after appropriate adjustment. However, K121Q did significantly influence the change in insulin sensitivity during lifestyle intervention after appropriate adjustment $\left(p_{\text {additive }}=0.0067, p_{\text {dominant }}=0.0027\right)$. Carriers of the minor allele had an impaired increase in OGTT-derived insulin sensitivity. A similar trend was obtained for clamp-derived insulin sensitivity, but did not reach significance.

Conclusions/interpretation In our population of European ancestry, the ENPP1 SNP K121Q influenced the change in insulin sensitivity during lifestyle intervention. Thus, this SNP may determine susceptibility to environmental changes and could predict the success of lifestyle intervention.

Keywords Ectoenzyme nucleotide pyrophosphatase phosphodiesterase $1 \cdot$ ENPP1 $\cdot$ PC1 Plasma cell membrane glycoprotein $1 \cdot$ SNP. Type 2 diabetes mellitus

\author{
Abbreviations \\ AU Arbitrary units \\ SNP Single nucleotide polymorphism
}

\section{Introduction}

Type 2 diabetes mellitus is a chronic progressive disease that results from impaired pancreatic beta cell function and peripheral and central insulin resistance [1]. Following its rapidly increasing incidence worldwide over the past decades, type 2 diabetes has reached epidemic proportions with major health consequences at an individual as well as a public health level [2]. Major environmental features of relevance to the development of type 2 diabetes are chronic 
overnutrition and a sedentary lifestyle against a background of genetic predisposition [3]. Lifestyle intervention programmes offer the possibility to delay or even prevent the onset of type 2 diabetes [4]. In light of limited healthcare resources, the identification of participants who would benefit particularly from lifestyle intervention is of key interest. Variations in different genes have been found to be associated with the success of a lifestyle intervention [5]. For instance, the rs2267668 A/G single nucleotide polymorphism (SNP) in the PPARD gene and the Gly482Ser SNP in the PPARGC1A gene impair the effectiveness with which aerobic exercise training increases aerobic physical fitness and insulin sensitivity [6].

Further interesting candidates are certainly the recently identified risk regions for type 2 diabetes. Given that improvement of insulin sensitivity is a major objective of lifestyle intervention [4], genetic variants associated with insulin sensitivity are of particular interest. However, while the majority of these risk alleles for type 2 diabetes appear to be associated with impaired pancreatic beta cell function, only a limited number of genetic variations, such as ENPP1 risk alleles, have been found to be associated with insulin resistance [7-12]. The ENPP1 gene, also known as $P C-1$, is located on the long arm of chromosome 6 (6q23.2) and encodes for a protein that interacts with the $\alpha$-subunit of the insulin receptor and inhibits subsequent signalling by reducing autophosphorylation of its $\beta$-subunit [13]. In vitro substitution of lysine by glutamine at codon 121 (K121Q) revealed a protein with a two- to threefold increased binding affinity for the insulin receptor and a more pronounced inhibition of the insulin receptor compared with the wild-type ectoenzyme nucleotide pyrophosphatase phosphodiesterase 1 protein $(\mathrm{K} 121 \mathrm{~K})$ [14]. In humans, the ENPP1 SNP K121Q (rs1044498) has shown positive association with insulin resistance $[15,16]$ and type 2 diabetes [17-22] in various ethnic populations, while a recent study showed that the association between ENPP1 K121Q and diabetes incidence was abolished by lifestyle modification [23]. However, results on the K121Q polymorphism are contradictory, as the positive association between K121Q and diabetes risk was not confirmed by all studies [19, 22, 24-26]. Therefore, in the present study, we aimed to broaden knowledge of a potential interaction between K121Q and lifestyle intervention, hypothesising that ENPP1 SNP K121Q may predict the outcome of lifestyle intervention.

\section{Methods}

Participants The 1,563 non-diabetic participants (characteristics, see Electronic supplementary material [ESM] Table 1) with a family history of type 2 diabetes or diagnosis of impaired fasting glycaemia were recruited from an ongoing study on the pathophysiology of type 2 diabetes [27]. The participants were not taking any medication known to affect glucose tolerance or insulin sensitivity. All participants were metabolically characterised by an OGTT. A subgroup of 506 participants was additionally characterised by a euglycaemic-hyperinsulinaemic clamp. We also analysed longitudinal data from a subgroup of 342 individuals who were at increased risk of type 2 diabetes (ESM Table 1) and were metabolically reanalysed after a follow-up period of 9 months (mean follow-up time $259 \pm 56$ [SD] days), during which they took part in a lifestyle intervention programme. Individuals were included in the interventional study if they fulfilled at least one of the following criteria: family history of type 2 diabetes, BMI $>27 \mathrm{~kg} / \mathrm{m}^{2}$, previous diagnosis of impaired glucose tolerance and/or of gestational diabetes.

This intervention study consisted of an exercise and dietary intervention adopted from that used in a previous study [28] and was designed to find variables that predict the effect of a lifestyle intervention in the form of diet and a moderate increase in aerobic physical activity geared to improving prediabetes phenotypes and the cardiovascular risk profile. The programme has been previously described in detail [29]. The participants aimed for a weight loss of at least $5 \%$, a reduction of energy intake from fat to less than $30 \%$ of total energy and an increase of fibre intake to at least $15 \mathrm{~g} / 4,184 \mathrm{~kJ}$ (1,000 kcal). They were also asked to reduce intake of saturated fat to less than $10 \%$ of fat intake. All individuals had up to ten sessions with a dietitian over a period of 9 months. During each visit, participants presented a 3-day food diary and discussed the results with the dietitians. Individuals were given a heart rate monitor (Polar, Büttelborn, Germany) and instructed to exercise while wearing this watch. This watch allowed us to document exercise intensity of the individuals. Individuals were asked to perform at least $3 \mathrm{~h}$ of moderate sports per week.

The participants gave informed written consent to the study. The protocol was approved by the Ethics Committee of the University of Tübingen.

Genotyping of the study population Study participants were genotyped for K121Q (rs1044498) in exon 4 of the ENPP1 gene, which was shown to increase the risk of type 2 diabetes in whites in a recent meta-analysis [12]. Genotyping was performed using the TaqMan assay, as previously described [27]. The overall genotyping success rate was $100 \%$, and re-screening of $3.2 \%$ of participants yielded $100 \%$ identical results.

OGTT At 08:00 hours participants ingested a solution containing $75 \mathrm{~g}$ glucose. Venous blood samples were obtained at $0,30,60,90$ and $120 \mathrm{~min}$ for determination 
of plasma glucose and insulin concentrations. Tests were performed after an overnight fast of $12 \mathrm{~h}$.

Euglycaemic-hyperinsulinaemic clamp After an overnight fast and drawing of baseline blood samples, a priming dose of insulin was given, followed by an infusion $\left(40 \mathrm{mU} / \mathrm{m}^{2}\right)$ of short-acting human insulin for $120 \mathrm{~min}$. A variable infusion of $20 \%$ glucose was started to maintain the plasma glucose concentration at the fasting level. Blood samples for the measurement of plasma glucose were obtained at $5 \mathrm{~min}$ intervals throughout the clamp. Plasma insulin levels were measured at baseline and in the steady state of the clamp.

Analytical procedures Blood glucose was determined using a bedside glucose analyser (glucose-oxidase method; Yellow Springs Instruments, Yellow Springs, OH, USA). Plasma insulin concentration was measured by a commercial chemiluminescence assay (ADVIA Centaur; Siemens Medical Solutions, Fernwald, Germany).

Body composition and body fat distribution Body mass index was calculated as weight $(\mathrm{kg})$ divided by height $(\mathrm{m})$ squared. Waist circumference was assessed in the standing position, midway between the highest point of the iliac crest and the lowest point of the costal margin in the midaxillary line.

Calculations Insulin sensitivity during the OGTT was estimated from glucose and insulin values as proposed by Matsuda and DeFronzo [30]. Insulin sensitivity during the euglycaemic-hyperinsulinaemic clamp was calculated by dividing the average glucose infusion rate during the last 40 min of the clamp steady state by the average plasma insulin concentration during the same time interval.

Statistical analyses Usually, data are given as means \pm SD. $\log _{e}$-transformation of metabolic variables was performed prior to simple and multivariate linear regression analyses. In multivariate linear regression models, the trait was chosen as dependent variable and sex, age, BMI and genotype were tested as independent variables. Changes in anthropometrics were adjusted for baseline values, sex and age. Changes in glucose levels and indices of insulin sensitivity were adjusted for baseline values, sex, age, BMI at baseline and changes in BMI. Adjustment of changes during lifestyle intervention comprised the respective baseline values to correct for potential ceiling effects. Covariates were included in the regression model on the basis of an a priori decision made upon ample epidemiological evidence $[28,31,32]$. To account for the three inheritance models and the two endpoints (anthropometrics and insulin sensitivity) tested, a Bonferroni corrected $\alpha$ level of $<0.0085$ was considered statistically significant.
The statistical software package JMP 7.0 (SAS Institute, Cary, NC, USA) was used. In the dominant inheritance model, the cross-sectional study was sufficiently powered $(1-\beta>0.8)$ to detect effect sizes $\geq 16 \%$ and the longitudinal study to detect effect sizes $\geq 39 \%$ (two-tailed $t$ test). Power calculation was performed using $\mathrm{G}^{*}$ power 3.0 software, available at www.psycho.uni-duesseldorf.de/aap/projects/ gpower (accessed 2 August 2009). Hardy-Weinberg equilibrium was tested using the $\chi^{2}$ test.

\section{Results}

ENPP1 SNP K121Q The HapMap and observed minor allele frequency for K121Q (Q allele) were 0.127 and 0.133 , respectively. The analysed polymorphism was in Hardy-Weinberg equilibrium $(p=0.9)$.

Cross-sectional analysis of associations of K121Q with anthropometric and metabolic data As shown in Table 1, K121Q was not associated with measures of obesity, indices of glucose tolerance during OGTT (e.g. fasting and $2 \mathrm{~h}$ glucose), insulin sensitivity estimated from the OGTT or insulin sensitivity derived from a euglycaemichyperinsulinaemic clamp after appropriate adjustment.

Longitudinal analysis of associations of K121Q with anthropometric and metabolic data during lifestyle intervention In 342 individuals, a 9-month lifestyle intervention was performed. In agreement with the overall cohort, at baseline K121Q was not associated with measures of obesity, indices of glucose tolerance during OGTT, insulin sensitivity estimated from the OGTT or insulin sensitivity derived from a euglycaemic-hyperinsulinaemic clamp after appropriate adjustment (ESM Table 2). After the intervention, BMI (before $30.1 \pm 5.6 \mathrm{~kg} / \mathrm{m}^{2}$, after $29.2 \pm 5.6 \mathrm{~kg} / \mathrm{m}^{2}, p<0.0001$ ), waist circumference (before $98 \pm 14 \mathrm{~cm}$, after $94 \pm 13 \mathrm{~cm}, p<0.0001)$ and OGTT-derived insulin sensitivity (before $12.7 \pm 6.9$ arbitrary units [AU], after $14.4 \pm 7.5 \mathrm{AU}, p<0.0001)$ were significantly improved. Clamp-derived insulin sensitivity, which was available in a limited number of participants $(n=57)$, tended to be improved (before $0.064 \pm 0.038 \mathrm{AU}$, after $0.072 \pm$ $0.047 \mathrm{AU}, p=0.09$ ). Post-intervention, K121Q was associated with OGTT-derived insulin sensitivity after adjustment for age, sex and BMI in the additive model $(p=0.022)$ (ESM Table 2). In the dominant model, K121Q tended to be associated with OGTT-derived insulin sensitivity after appropriate adjustment $(p=0.07)$.

As shown in Fig. 1, the minor 121Q minor allele was significantly associated with an impaired increase in OGTTderived insulin sensitivity after lifestyle intervention fol- 
Table 1 Cross-sectional analysis of ENPP1 SNP K121Q (rs1044498) effects in the overall cohort $(n=1,563)$

\begin{tabular}{|c|c|c|c|c|c|c|}
\hline \multirow[t]{2}{*}{ Characteristics } & \multicolumn{3}{|l|}{ Genotype } & \multicolumn{3}{|c|}{$p$ value } \\
\hline & KK & KQ & QQ & $p_{\text {add }}$ & $p_{\text {dom }}$ & $p_{\text {rec }}$ \\
\hline$n$ & 1,174 & 363 & 26 & - & - & - \\
\hline Age (years) & $40 \pm 13$ & $39 \pm 13$ & $42 \pm 15$ & 0.3 & 0.4 & 0.3 \\
\hline BMI $\left(\mathrm{kg} / \mathrm{m}^{2}\right)$ & $28.9 \pm 8.4$ & $28.5 \pm 7.7$ & $28.4 \pm 5.7$ & 0.9 & 0.7 & 0.9 \\
\hline Waist circumference $(\mathrm{cm})$ & $94 \pm 18$ & $93 \pm 16$ & $93 \pm 12$ & 0.9 & 0.7 & 0.9 \\
\hline Fasting glucose $(\mathrm{mmol} / \mathrm{l})$ & $5.11 \pm 0.54$ & $5.08 \pm 0.57$ & $5.21 \pm 0.45$ & 0.4 & 0.4 & 0.4 \\
\hline $120 \mathrm{~min}$ glucose $(\mathrm{mmol} / \mathrm{l})$ & $6.29 \pm 1.68$ & $6.20 \pm 1.58$ & $6.47 \pm 1.54$ & 0.8 & 0.9 & 0.6 \\
\hline ISI, OGTT (AU) & $16.2 \pm 10.6$ & $16.7 \pm 10.9$ & $15.7 \pm 12.8$ & 0.6 & 0.7 & 0.4 \\
\hline ISI, clamp (AU) ${ }^{\mathrm{a}}$ & $0.084 \pm 0.054$ & $0.092 \pm 0.060$ & $0.053 \pm 0.028$ & 0.07 & 0.2 & 0.11 \\
\hline
\end{tabular}

Values are means $\pm \mathrm{SD}$

For statistical analysis, data were $\log _{e}$-transformed. Anthropometric data were adjusted for sex and age. Plasma glucose levels and indices of insulin sensitivity were adjusted for sex, age and BMI

${ }^{a}$ Data available from 506 participants

ISI, insulin sensitivity index; $p_{\text {add, }} p$ value additive model; $p_{\text {dom, }} p$ value dominant model; $p_{\text {rec, }} p$ value recessive model

lowing adjustment for insulin sensitivity at baseline, sex, age, BMI at baseline and changes in BMI. The impact of the covariates on change of insulin sensitivity is given in ESM Table 3.

In a subsequent analysis, the model was modified to replace the carrier variable with a continuous variable equal to 0 for major allele homozygotes, 1 for heterozygotes and 2 for minor allele homozygotes. This model was only used to test for linear trend associated with allele dose. In this analysis, too, K121Q was significantly associated with

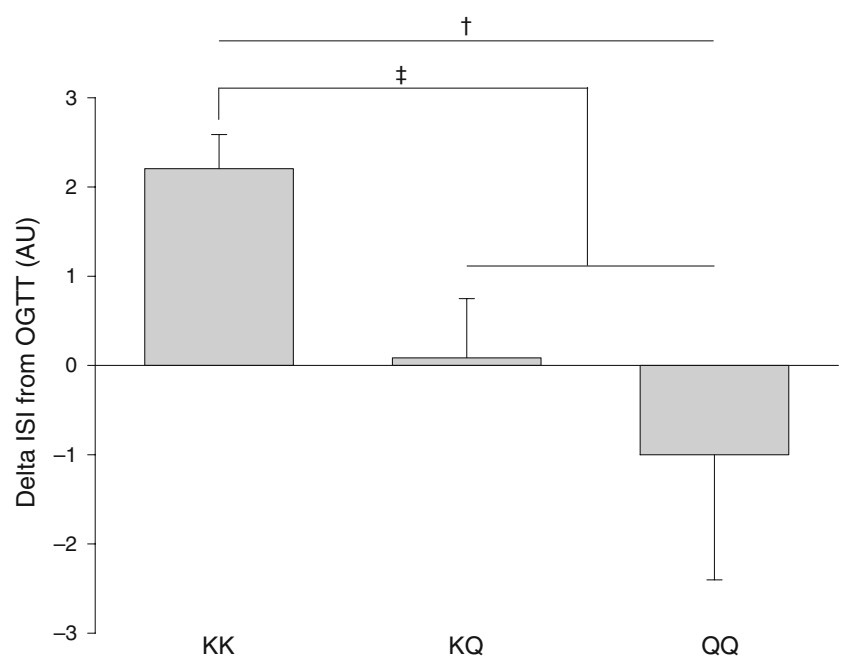

Fig. 1 Impact of ENPP1 SNP K121Q on changes in insulin sensitivity index (ISI) during lifestyle intervention. Unadjusted data from 342 participants are presented. For statistical reasons, data were $\log _{e}$-transformed, adjusted for insulin sensitivity at baseline, sex, age, $\mathrm{BMI}$ at baseline and changes in BMI, and analysed in the additive and dominant models. ${ }^{\dagger} p=0.0007,{ }^{\dagger} p=0.0027$ change in OGTT-derived insulin sensitivity after lifestyle intervention following adjustment for insulin sensitivity at baseline, sex, age, BMI at baseline and changes in BMI ( $p=$ 0.0016).

Carriers of the diabetes risk allele displayed decreased clamp-derived insulin sensitivity after adjustment for insulin sensitivity at baseline, sex, age, BMI at baseline and changes in BMI in the dominant model. However, carriers of the $\mathrm{K}$ allele showed increased clamp-derived insulin sensitivity, a difference that tended towards significance (AA $0.012 \pm 0.027 \mathrm{AU}$ vs XC $-0.016 \pm 0.019 \mathrm{AU}, p=$ 0.09) (ESM Table 2). In contrast, intervention-induced changes in measures of obesity and indices of glucose tolerance during OGTT were not associated with K121Q after appropriate adjustment ( $p>0.14$ for all) (ESM Table 2).

\section{Discussion}

In the present study, we aimed to investigate the impact of the K121Q SNP in the ENPP1 gene, which was reported to be associated with type 2 diabetes in a recent meta-analysis [12], on the success of a lifestyle intervention programme in individuals at increased risk of type 2 diabetes.

We found a significant impact of K121Q on change in insulin sensitivity during lifestyle intervention. Minor allele carriers had an impaired increase in OGTT-derived insulin sensitivity after lifestyle intervention. Similar results were obtained for clamp-derived insulin sensitivity, but did not reach significance, most probably due to the limited number of participants investigated by this technique. 
In contrast, we did not find an association between K121Q and insulin sensitivity in our cross-sectional study. In non-diabetic participants, associations between the $121 \mathrm{Q}$ allele and insulin resistance have been described in many [15-21] but not in all [17, 18, 24] populations. Similarly, K121Q was associated with type 2 diabetes in various ethnic groups, including whites living in the USA [17, 18] and Finland [20], but not in whites of European ancestry in Sweden [19], Denmark [22, 24], UK [25] and the USA or Poland [26]. Though currently unknown, the discrepant effects of the 121Q allele on metabolic traits in different populations may result from modification by environmental factors or from functional genetic variations in other genes [33], which may differ between populations.

While our data suggest an impact of K121Q on change in insulin sensitivity at least during a 9-month lifestyle intervention, a recent study showed that the association between 121Q and diabetes incidence was abolished by lifestyle modification [23]. The reasons for this discrepancy are currently unclear, but may be due to the following differences in the population characteristics: (1) percentage of participants with impaired glucose tolerance (previous study [23] 100\%, our study 27\%); (2) ethnicity (previous study [23] 55\% whites, our study $100 \%$ whites); (3) age (previous study [23] 51 years, our study 46 years); and (4) BMI (previous study [23] $34 \mathrm{~kg} / \mathrm{m}^{2}$, our study $30 \mathrm{~kg} / \mathrm{m}^{2}$ ). Given that the majority of participants in our study as well as in that by Moore et al. [23] were overweight or had grade 1 obesity and were therefore at moderate risk of type 2 diabetes, it would be interesting for future studies to investigate the impact of K121Q on lifestyle intervention in severely obese participants at high risk of type 2 diabetes.

The present study has certain limitations. First, further replications in other cohorts are clearly needed to confirm the impact of genetic variation within this gene on changes in insulin sensitivity during lifestyle intervention, especially as no control group was subjected to follow-up. Second, we were not able to detect effect sizes smaller than $39 \%$ with sufficient power $(80 \%)$, e.g. in changes in weight-related traits and in both fasting and 2-h glycaemia during lifestyle modification. Consequently small to moderate effects of K121Q on these traits may have remained undetected and the associations with weight-related traits and glycaemia need to be reassessed in larger lifestyle intervention studies. Third, $p$ values of the significant findings were marginal not only from a whole-genome perspective, but also in terms of general concerns about reproducibility commonly seen in exploratory genetic association studies. However, our study was hypothesis-driven and our $p$ values withstood Bonferroni correction for the three inheritance models and the two endpoints (anthropometrics and insulin sensitivity) tested.

In conclusion, in our population of European ancestry at increased risk for type 2 diabetes, ENPP1 SNP K121Q was associated with changes in insulin sensitivity during lifestyle intervention. Thus this SNP may determine susceptibility to environmental changes and could predict the success of lifestyle intervention in high-risk patients.

Acknowledgements We thank all study participants for their cooperation. We thank the International HapMap Consortium for the public allocation of genotype data. We gratefully acknowledge the excellent technical assistance of A. Bury, A. Guirguis, H. Luz, M. Weisser and R. Werner. The study was supported in part by grants from the German Research Foundation (KFO 114/2) and the European Community's frame programme FP6 EUGENE 2 (LSHM-CT-2004-512013).

Duality of interest The authors declare that there is no duality of interest associated with this manuscript.

\section{References}

1. Kahn SE (2003) The relative contributions of insulin resistance and beta-cell dysfunction to the pathophysiology of type 2 diabetes. Diabetologia 46:3-19

2. Wild S, Roglic G, Green A, Sicree R, King H (2004) Global prevalence of diabetes: estimates for the year 2000 and projections for 2030. Diabetes Care 27:1047-1053

3. Lieberman LS (2003) Dietary, evolutionary, and modernizing influences on the prevalence of type 2 diabetes. Annu Rev Nutr 23:345-377

4. Schulze MB, Hu FB (2005) Primary prevention of diabetes: what can be done and how much can be prevented? Annu Rev Public Health 26:445-467

5. Weyrich P, Stefan N, Häring HU, Laakso M, Fritsche A (2007) Effect of genotype on success of lifestyle intervention in subjects at risk for type 2 diabetes. J Mol Med 85:107-117

6. Stefan N, Thamer C, Staiger $\mathrm{H}$ et al (2007) Genetic variations in PPARD and PPARGC1A determine mitochondrial function and change in aerobic physical fitness and insulin sensitivity during lifestyle intervention. J Clin Endocrinol Metab 92:1827-1833

7. Sladek R, Rocheleau G, Rung J et al (2007) A genome-wide association study identifies novel risk loci for type 2 diabetes. Nature 445:881-885

8. Zeggini E, Weedon MN, Lindgren CM et al (2007) Replication of genome-wide association signals in UK samples reveals risk loci for type 2 diabetes. Science 316:1336-1341

9. Saxena R, Voight BF, Lyssenko V et al (2007) Genome-wide association analysis identifies loci for type 2 diabetes and triglyceride levels. Science 316:1331-1336

10. Unoki H, Takahashi A, Kawaguchi $\mathrm{T}$ et al (2008) SNPs in KCNQ1 are associated with susceptibility to type 2 diabetes in East Asian and European populations. Nat Genet 40:1098-1102

11. Yasuda K, Miyake K, Horikawa Y et al (2008) Variants in KCNQ1 are associated with susceptibility to type 2 diabetes mellitus. Nat Genet 40:1092-1097

12. McAteer JB, Prudente S, Bacci S et al (2008) The ENPP1 K121Q polymorphism is associated with type 2 diabetes in European populations: evidence from an updated meta-analysis in 42, 042 subjects. Diabetes 57:1125-1130

13. Maddux BA, Goldfine ID (2000) Membrane glycoprotein PC-1 inhibition of insulin receptor function occurs via direct interaction with the receptor alpha-subunit. Diabetes 49:13-19

14. Costanzo BV, Trischitta V, Di Paola R et al (2001) The Q allele variant (GLN121) of membrane glycoprotein PC-1 interacts with the insulin receptor and inhibits insulin signaling more effectively than the common K allele variant (LYS121). Diabetes 50:831-836 
15. Kubaszek A, Pihlajamaki J, Karhapaa P, Vauhkonen I, Laakso M (2003) The K121Q polymorphism of the PC-1 gene is associated with insulin resistance but not with dyslipidemia. Diabetes Care 26:464-467

16. Pizzuti A, Frittitta L, Argiolas A et al (1999) A polymorphism (K121Q) of the human glycoprotein PC-1 gene coding region is strongly associated with insulin resistance. Diabetes 48:18811884

17. Abate N, Carulli L, Cabo-Chan A Jr, Chandalia M, Snell PG, Grundy SM (2003) Genetic polymorphism PC-1 K121Q and ethnic susceptibility to insulin resistance. J Clin Endocrinol Metab 88:5927-5934

18. Abate N, Chandalia M, Satija P et al (2005) ENPP1/PC-1 K121Q polymorphism and genetic susceptibility to type 2 diabetes. Diabetes 54:1207-1213

19. Gu HF, Almgren P, Lindholm E et al (2000) Association between the human glycoprotein PC-1 gene and elevated glucose and insulin levels in a paired-sibling analysis. Diabetes 49:1601-1603

20. Kubaszek A, Markkanen A, Eriksson JG et al (2004) The association of the K121Q polymorphism of the plasma cell glycoprotein-1 gene with type 2 diabetes and hypertension depends on size at birth. J Clin Endocrinol Metab 89:2044-2047

21. Meyre D, Bouatia-Naji N, Tounian A et al (2005) Variants of ENPP1 are associated with childhood and adult obesity and increase the risk of glucose intolerance and type 2 diabetes. Nat Genet 37:863-867

22. Grarup N, Urhammer SA, Ek J et al (2006) Studies of the relationship between the ENPP1 K121Q polymorphism and type 2 diabetes, insulin resistance and obesity in 7, 333 Danish white subjects. Diabetologia 49:2097-2104

23. Moore AF, Jablonski KA, Mason CC et al (2009) The association of ENPP1 K121Q with diabetes incidence is abolished by lifestyle modification in the diabetes prevention program. J Clin Endocrinol Metab 94:449-455
24. Rasmussen SK, Urhammer SA, Pizzuti A et al (2000) The K121Q variant of the human PC-1 gene is not associated with insulin resistance or type 2 diabetes among Danish Caucasians. Diabetes 49:1608-1611

25. Weedon MN, Shields B, Hitman G et al (2006) No evidence of association of ENPP1 variants with type 2 diabetes or obesity in a study of 8, 089 U.K. Caucasians. Diabetes 55:3175-3179

26. Lyon HN, Florez JC, Bersaglieri T et al (2006) Common variants in the ENPP1 gene are not reproducibly associated with diabetes or obesity. Diabetes 55:3180-3184

27. Stefan N, Machicao F, Staiger H et al (2005) Polymorphisms in the gene encoding adiponectin receptor 1 are associated with insulin resistance and high liver fat. Diabetologia 48:2282-2291

28. Tuomilehto J, Lindstrom J, Eriksson JG et al (2001) Prevention of type 2 diabetes mellitus by changes in lifestyle among subjects with impaired glucose tolerance. N Engl J Med 344:1343-1350

29. Schäfer S, Kantartzis K, Machann J et al (2007) Lifestyle intervention in individuals with normal versus impaired glucose tolerance. Eur J Clin Invest 37:535-543

30. Matsuda M, DeFronzo RA (1999) Insulin sensitivity indices obtained from oral glucose tolerance testing: comparison with the euglycemic insulin clamp. Diabetes Care 22:1462-1470

31. Ma X, Becker D, Arena VC, Vicini P, Greenbaum C (2009) The effect of age on insulin sensitivity and insulin secretion in firstdegree relatives of type 1 diabetic patients: a population analysis. J Clin Endocrinol Metab 94:2446-2451

32. Masharani U, Goldfine ID, Youngren JF (2009) Influence of gender on the relationship between insulin sensitivity, adiposity, and plasma lipids in lean nondiabetic subjects. Metabolism 58:1602-1608

33. Baratta R, Di Paola R, Spampinato D et al (2003) Evidence for genetic epistasis in human insulin resistance: the combined effect of PC-1 (K121Q) and PPARgamma2 (P12A) polymorphisms. J Mol Med 81:718-723 